\title{
Gastric schwannoma misdiagnosed as GIST: A case report with immunohistochemical and molecular study
}

\author{
FABIANA TATANGELO ${ }^{1}$, MONICA CANTILE $^{1}$, FRANCESCA COLLINA $^{1}$, ANDREA BELLI $^{2}$, \\ SILVIA DE FRANCISCIS ${ }^{2}$, FRANCO BIANCO ${ }^{2}$ and GERARDO BOTTI ${ }^{1}$ \\ ${ }^{1}$ Pathology Unit; ${ }^{2}$ Department of General and Hepato-Pancreato-Biliary Surgery, National \\ Cancer Institute ‘G. Pascale' Foundation, Naples, Campania 80131, Italy
}

Received November 6, 2014; Accepted May 26, 2015

DOI: 10.3892/ol.2016.4281

\begin{abstract}
Schwannomas are tumors derived from Schwann cells. Generally, they are benign and their typical site of origin is the subcutaneous tissue of the distal extremities or of the head and neck region. Gastrointestinal localization of schwannomas is extremely rare, and the stomach is the prevalent site. The present study describes the case of a gastric schwannoma in a 61-year-old male who underwent subtotal gastrectomy following a clinical diagnosis of a gastrointestinal stromal tumor (GIST). A histological, immunohistochemical and molecular study was performed to exclude the misdiagnosis of GIST. The histomorphological features of the lesion and absence of c-Kit and PDGFRA mutations indicated the diagnosis of gastric schwannoma.
\end{abstract}

\section{Introduction}

Schwannomas are tumors derived from Schwann cells, generally affecting the subcutaneous tissue of the distal extremities and/or the head and neck region. Schwannomas are generally benign neoplasias, and are extremely rare in the gastrointestinal tract (1).

The stomach is the most common site for schwannomas in the gastrointestinal tract, occurring primarily in the gastric submucosa, followed by the colon $(2,3)$. The most infrequently affected sites are the small intestine and esophagus $(4,5)$.

The peculiar morphology and localization of gastric schwannomas often causes problems in the differential diagnosis between gastrointestinal stromal tumors (GISTs) and other mesenchymal tumors typically located in the stomach.

Correspondence to: Dr Monica Cantile, Pathology Unit, National Cancer Institute 'G. Pascale' Foundation, 1 via Mariano Semmola, Naples, Campania 80131, Italy

E-mail: monica.cantile@libero.it

Key words: gastric schwannoma, immunohistochemical and molecular study, gastrointestinal stromal tumor
The typical histological features of gastric schwannoma consist of focally atypical spindle cells that are usually arranged in a microtrabecular-microfascicular pattern and a peritumoral lymphoid cuff, often with germinal centers. S100 protein positivity, a frequent but variable immunoreactivity for GFAP, and negativity for c-Kit and smooth muscle markers have been described in immunohistochemical studies $(6,7)$. For the differential diagnosis of gastric schwannomas from GISTs, the determination of certain genetic characteristics, such as a lack of c-Kit and PDGFRA mutations (8) are extremely useful. For the differential diagnosis from soft-tissue schwannomas the analysis of somatic NF2 gene mutations, which are common in sporadic soft-tissue schwannomas, but rare in gastric schwannomas, is necessary (9). The genetic features of gastric schwannoma also include chromosome 22 monosomy and polyploidy of chromosome 2 and 18 (10).

Gastric schwannoma appears to exhibit a good prognosis, with no recurrence or metastasis, and previous follow-up studies have not found any malignant variants (11).

The present study describes the case of a 61-year-old male who presented with a clinical diagnosis of a GIST, and was subsequently diagnosed with gastric schwannoma following morphological, immunohistochemical and molecular investigations.

\section{Case report}

A 61-year-old Caucasian male, without comorbidities, but affected by dyspepsia and symptoms associated with gastro esophageal reflux disease, underwent a diagnostic esophagogastroduodenoscopy (EGD) revealing a $4-\mathrm{cm}$ bulky mass of the gastric antrum and a $3-\mathrm{cm}$ mass of the first portion of the duodenum. Endoscopic biopsies of the two lesions were inconclusive. In May 2012, the patient was admitted to INT Fondazione Pascale Hospital (Naples, Italy) and underwent a new EGD with endoscopic ultrasound, which highlighted a unique sub-mucosal heteroplasia protruding into the gastric antrum and duodenal bulb, with images suggestive of a diagnosis of a GIST. Blood tests including CEA, CA19-9, chromogranin, gastrin and NSE (Neuron Specific Enolase) were within normal values. A total body CT scan confirmed the presence of aforementioned lesion with images suggestive of infiltration of perigastric fat and evidence of regional lymph- 


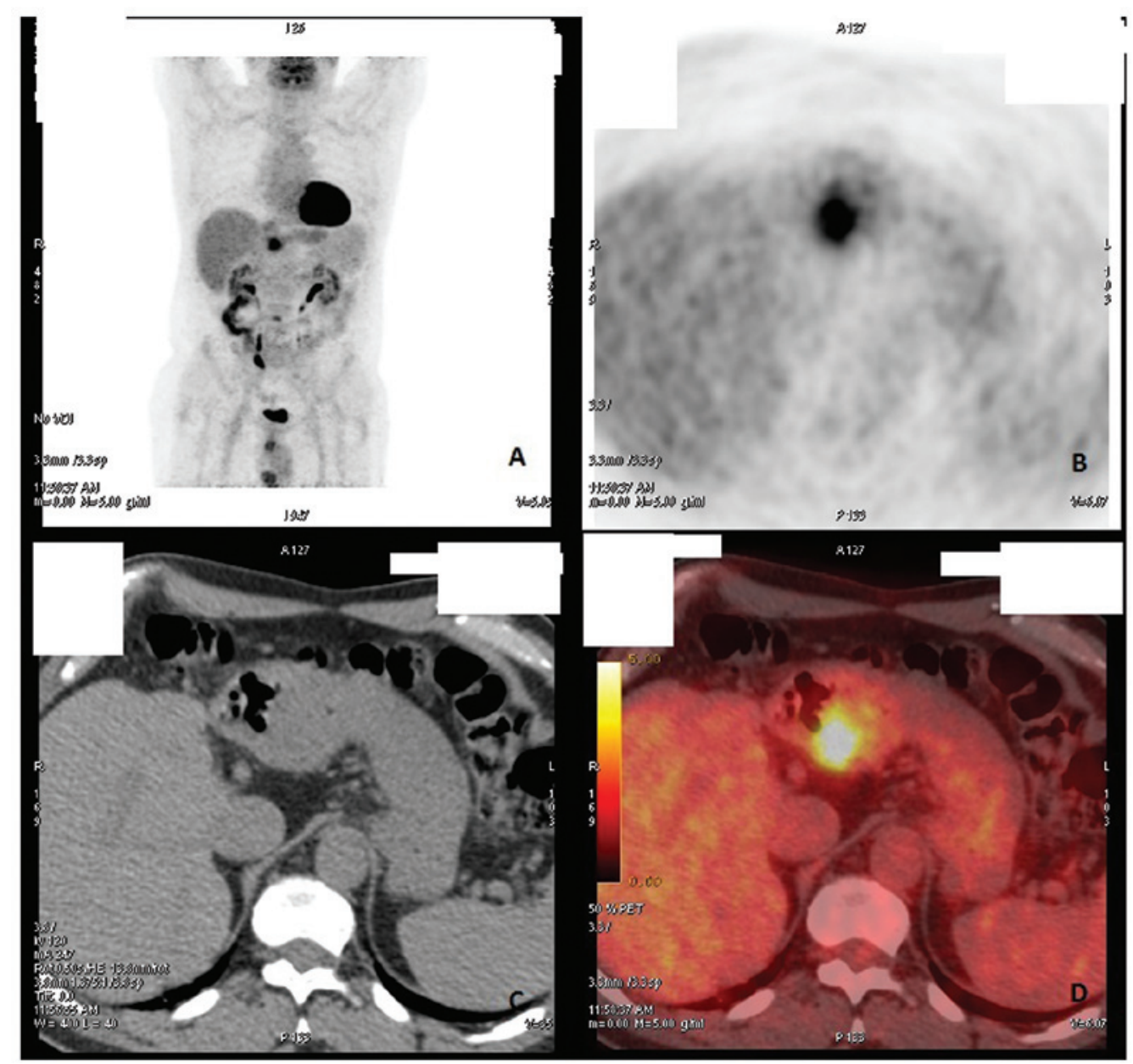

Figure 1. (A) Total body positron emission tomography-computed tomography (PET-CT) and (B) enlargement of the lesion in the axial position (C) Contrast-enhanced CT and (D) PET-CT showing a round, well-defined and homogeneously enhancing mass in the gastric antrum and duodenal bulb.
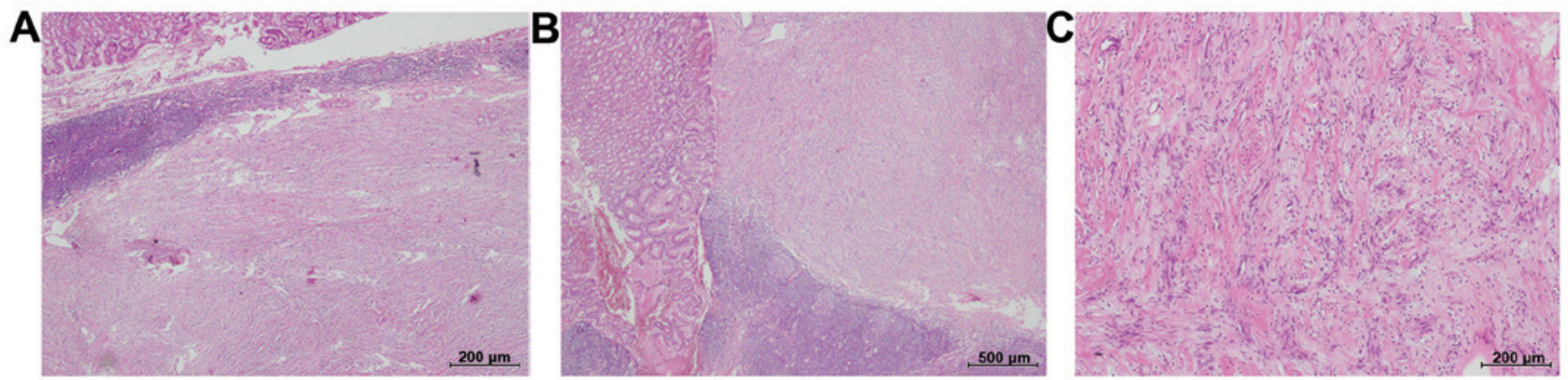

Figure 2. Gastric schwannoma sample. (A) H\&E-stained image with representative mucosa (top), crown lymphocytes and tumor (bottom) (x4 magnification); (B) H\&E-stained image with gastric mucosa (left), tumor (right) with a peritumoral lymphocytes cuff (x4 magnification); and (C) H\&E-stained image representative of typical fascicular pattern (x10 magnification). H\&E, hematoxylin and eosin.

adenopathies (Fig. 1). The patient underwent to a subtotal gastrectomy with D2 lymphadenectomy. The post-operative course was uneventful, with the patient discharged on post-operative day 7.

The surgery constituted of subtotal omento-gastrectomy, with stomach of $16 \times 13 \mathrm{~cm}$ (great curvature $\mathrm{x}$ lesser curvature) and omentum of $50 \times 38 \mathrm{~cm}$.

At the gastric corpus, an exophytic, round lesion $(3.5 \mathrm{~cm}$ in maximum diameter) was present covered by normal mucosa, with a fasciculated section surface. The omentum was macroscopically normal.

Under microscopic observation, the lesion was mainly composed of spindle cells nests and cords, and rare epithelioid cells, with a fasciculated/storiform growth pattern; the cytoplasm was eosinophilic, and the nuclei were dark and occasionally polymorphic (Fig. 2). Mitotic activity was almost absent ( $<5 / 50$ high-power fields). The $\mathrm{Ki}-67$ proliferation index was low (3\%) and cellular atypia was absent. 


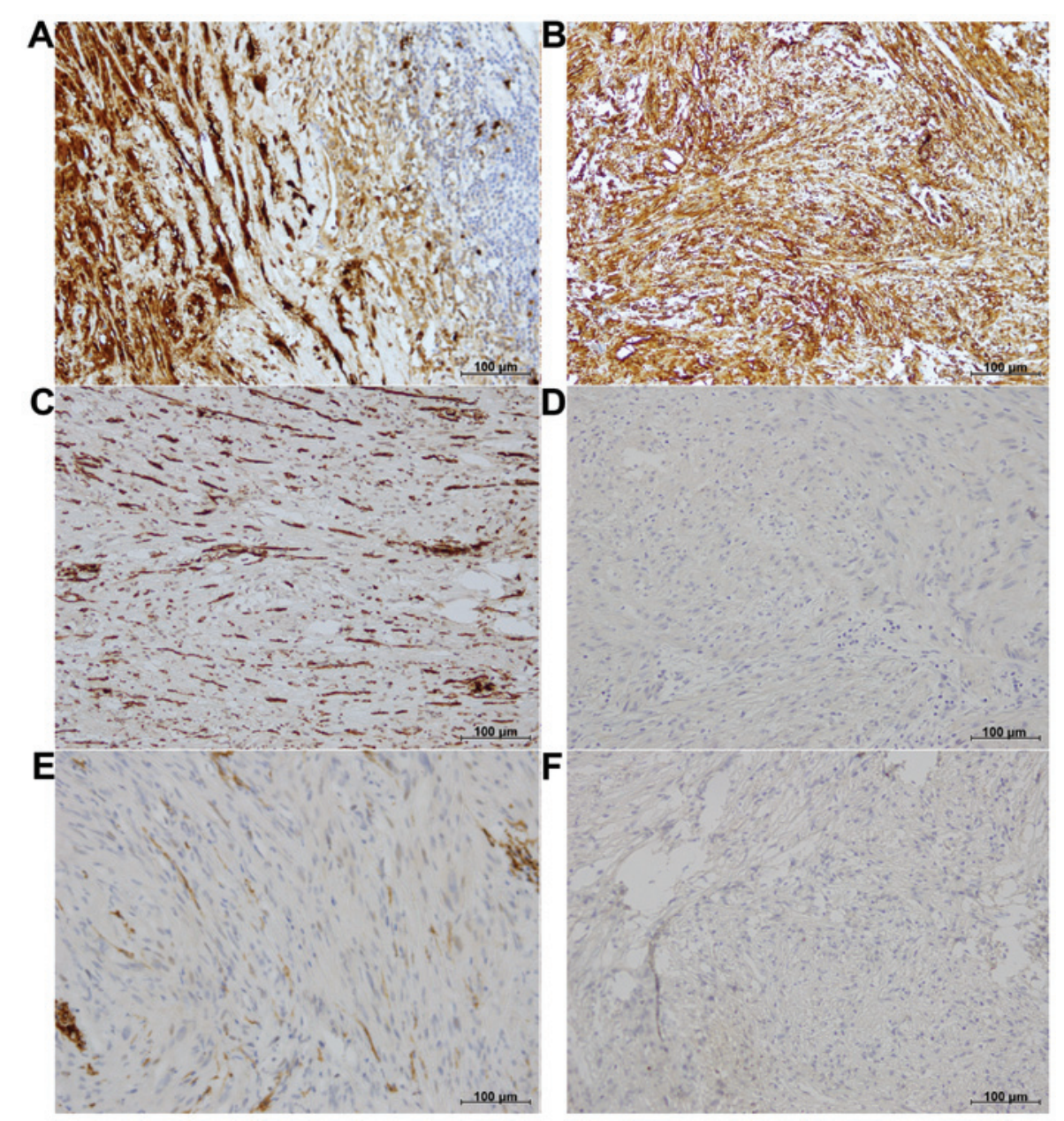

Figure 3. Gastric schwannoma immunoprofile. Positive immunostaining for (A) S100 (x20 magnification) and (B) vimentin (x20 magnification); and negative immunostaining for (C) actin (x20 magnification), (D) desmin (x20 magnification), (E) cluster of differentiation 34 (x20 magnification) and (F) c-Kit (x20 magnification).

The neoplastic proliferation affected the sub-mucosal layers and was covered by normal gastric mucosa.

The lesion showed a peripheral covering of small lymphocytes confluent in lymphoid aggregates with a few germinal centers.

The panel of IHC markers used showed a negative cellular reaction for cluster of differentiation (CD)117/c-Kit (CD117, 1:50; Dako, Carpinteria, CA, USA), CD34 (QBEnd/10, 1:40; BioGenex, San Ramon, CA, USA), SMA (1A4, 1:800; Sigma Chemicals, St. Louis, MO, USA) and desmin (D33, 1:40; Dako) (Fig. 3).

A moderate positive cellular reaction for vimentin (D9, 1:1,000; NeoMarkers, Westinghouse, CA, USA) and a strong cellular reaction for S100 (1:3,000; Dako) were present (Fig. 3).

The microscopic features, particularly with respect to the pattern of growth and the presence of peripheral lymphoid tissue with germinal centers, and the association with the immunohistochemical features, were conclusive for a final histological diagnosis of a gastric tumor with Schwann cells or a gastric schwannoma.

Finally, following the histological diagnosis, in order to completely solve the problem of the differential diagnosis with a GIST, a mutational analysis of the c-Kit and PDGFRA genes was performed. A representative formalin-fixed, paraffin- embedded (FFPE) sample of the case was selected and following DNA purification (QIAamp DNA FFPE Tissue kit; Qiagen, Valencia, CA, USA), polymerase chain reaction (PCR) was performed on 25-50 ng of isolated genomic DNA in a 9700 Thermal Cycler (Applied Biosystems, Foster City, CA, USA). All PCR-amplified products were directly sequenced using an automated fluorescence-cycle sequencer (ABI PRISM 3130; Applied Biosystems). No c-Kit or PDGFRA mutations were detected in the sample (Fig. 4).

Written informed consent was obtained from the patient for publication of this study and the accompanying images. Currently the patient is well and is still undergoing follow-up at INT Fondazione Pascale Hospital.

\section{Discussion}

Gastric schwannomas are rare stromal tumors that consist of spindle cells of the gastrointestinal tract and arise from the Schwann cells of the gastrointestinal neural plexus. The stomach is the preferential site of localization and schwannomas represent $\sim 0.2 \%$ of all gastric neoplasms (12).

The differential diagnosis for this neoplasm is difficult due to the macroscopic and microscopic similarities with other typical lesions of the stomach. In particular, the pres- 


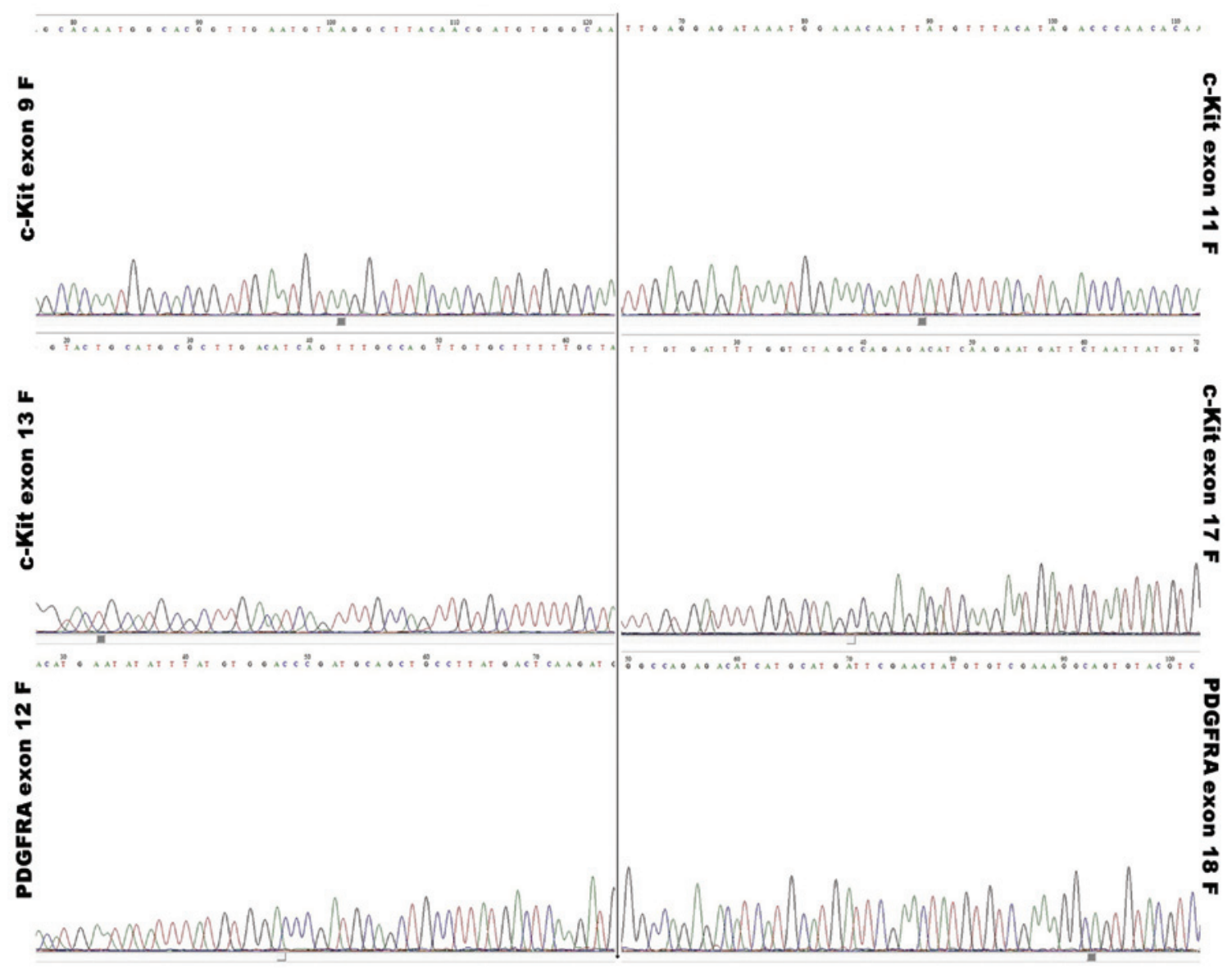

Figure 4. c-Kit and PDGFRA gene mutational analysis. DNA sequencing electropherograms showing an absence of mutations in exons $9,11,13$ and 17 of the c-Kit gene, and in exons 12 and 18 of the PDGFRA gene.

ence of spindle cells that are slow growing and covered with normal mucosa is common to a large series of gastric tumors defined generically as 'spindle cell tumors of the gastrointestinal tract' (13).

However, immunohistochemical staining is useful in these cases, as S100 protein staining can be applied for differentiating schwannomas from other spindle cell tumors of the stomach (7). Generally, S100 protein-positive and smooth muscle actin- and c-Kit-negative tumor cells support the diagnosis of a schwannoma (11).

The patient in the present study came to our attention due to a presumptive diagnosis of a GIST, based solely on radiographic and endoscopic investigations. The tumor sample exhibited sub-mucosal layers, covered by normal gastric mucosa, and was morphologically characterized by spindle cells nests and cords, and rare epithelioid cells, with a fasciculated/storiform growth pattern.

An immunohistochemical study was performed to correctly define the histomorphological features of the lesion. The IHC panel showed strong positivity for S100 and negativity for c-Kit, CD34, SMA and desmin. These observations alone could be sufficient to exclude a diagnosis of GIST, however, GISTs are also characterized by several activating mutations in the c-Kit and PDGFRA genes (8). The majority of the mutations are located in the juxtamembrane domain (exon 11), followed by the extracellular domain (exon 9) and less commonly, the kinase domains (exon 13 and 17). The most common mutations in PDGFRA are located in exons 12,14 and 18. c-Kit and PDGFRA mutations appear to be mutually exclusive oncogenic events in GISTs (8).

For this reason, a mutational analysis was performed in the present study to detect c-Kit and PDGFRA mutations in the tumor sample, but no gene alterations were found.

Therefore, molecular analysis appears to be extremely useful, even if it is more expensive than in situ analyses and the response times are much longer. In any case, the IHC evaluation may be indicative of the presence of activating mutations of the two genes.

However, a previous study showed that in a small percentage of GISTs, immunohistochemistry was negative for c-Kit protein staining, negative for desmin and S100 protein in the majority of cases, and positive for smooth muscle actin in $\sim 50 \%$ of cases (14).

The present IHC panel and the molecular analysis supported the gastric schwannoma diagnosis. The correct diagnosis is also crucial in order to determine the correct therapeutic strategies. In fact, while gastric schwannoma has a good prognosis and the post-operative prognosis is excellent, recurrent disease is in fact generally associated only with an incomplete surgical margin. The prognosis of patients with GISTs is poor owing to the frequent recurrence, and the resistance to chemotherapy and radiotherapy regimens (15). GISTs with several point mutations in the c-Kit gene may benefit from the use of specific 
receptor tyrosine kinase inhibitors, such as imatinib mesylate (Gleevec) (15).

In conclusion, the correct histomorphological revision, supported by specific IHC panel and molecular investigations, to exclude in particular the diagnosis of GISTs, is fundamental, as misdiagnosis may prevent the patient from benefiting from specific target therapies.

\section{References}

1. Melvin WS and Wilkinson MG: Gastric schwannoma. Clinical and pathologic considerations. Am Surg 59: 293-296, 1993.

2. Braumann C, Guenther N, Menenakos C and Junghans T: Schwannoma of the colon mimicking carcinoma: A case report and literature review. Int J Colorectal Dis 22: 1547-1548, 2007.

3. Jacobson BC, Hirsch MS, Lee JH, Van Dam J, Shoji B and Farraye FA: Multiple asymptomatic plexiform schwannomas of the sigmoid colon: A case report and review. Gastrointest Endosc 53: 801-804, 2001

4. Tokunaga T, Takeda S, Sumimura J and Maeda H: Esophageal schwannoma: Report of a case. Surg Today 37: 500-502, 2007.

5. Kitada M, Matsuda Y, Hayashi S, Ishibashi K, Oikawa K and Miyokawa N: Esophageal schwannoma: A case report. World J Surg Oncol 11: 253, 2013.

6. Hirose T, Ishizawa K, Sakaki M and Fujii Y: Retroperitoneal schwannoma is characterized by a high incidence of cellular type and GFAP-immunoreactivity. Pathol Int 62: 456-462, 2012.
7. Sarlomo-Rikala M and Miettinen M: Gastric schwannoma - a clinicopathological analysis of six cases. Histopathology 27: 355-360, 1995.

8. Lasota $J$ and Miettinen M: Clinical significance of oncogenic KIT and PDGFRA mutations in gastrointestinal stromal tumours. Histopathology 53: 245-266, 2008.

9. Ogasawara N, Sasaki M, Ishiguro H, Itoh Y, Nojiri S, Kubota E, Wada T, Kataoka H, Kuwabara Y and Joh T: Gastric schwannoma with adjacent external progression harbored aberrant NF2 gene. Dig Endosc 21: 192-195, 2009.

10. Díaz de Ståhl T, Hansson CM, de Bustos C, Mantripragada KK, Piotrowski A, Benetkiewicz M, Jarbo C, Wiklund L, Mathiesen T, Nyberg G, et al: High-resolution array-CGH profiling of germline and tumor-specific copy number alterations on chromosome 22 in patients affected with schwannomas. Hum Genet 118: 35-44, 2005.

11. Voltaggio L, Murray R, Lasota J and Miettinen M: Gastric schwannoma: A clinicopathologic study of 51 cases and critical review of the literature. Hum Pathol 43: 650-659, 2012.

12. Snyder RA, Harris E, Hansen EN, Merchant NB and Parikh AA: Gastric schwannoma. Am Surg 74: 753-756, 2008.

13. Miettinen $M$ and Lasota J: Histopathology of gastrointestinal stromal tumor. J Surg Oncol 104: 865-873, 2011.

14. Miettinen M, Sobin LH and Sarlomo-Rikala M: Immunohistochemical spectrum of GISTs at different sites and their differential diagnosis with a reference to CD117 (KIT). Mod Pathol 13: 1134-1142, 2000.

15. Papaetis GS and Syrigos KN: Targeted therapy for gastrointestinal stromal tumors: Current status and future perspectives. Cancer Metastasis Rev 29: 151-170, 2010. 